\title{
A Newton-Krylov Solver for Fast Spin-up of Online Ocean Tracers
}

\author{
Keith Lindsay \\ National Center for Atmospheric Research, P.O. Box 3000, Boulder, CO 80307-3000, \\ $U S A$
}

\section{Abstract}

We present a Newton-Krylov based solver to efficiently spin up tracers in an online ocean model. We demonstrate that the solver converges, that tracer simulations initialized with the solution from the solver have small drift, and that the solver takes orders of magnitude less computational time than the brute force spin-up approach. To demonstrate the application of the solver, we use it to efficiently spin up the tracer ideal age with respect to the circulation from different time intervals in a long physics run. We then evaluate how the spun-up ideal age tracer depends on the duration of the physics run, i.e., on how equilibrated the circulation is.

Keywords: Newton-Krylov, Tracers, Ideal Age, Ocean Modelling

\section{Introduction}

2 Analysis of ocean tracer simulations is complicated by the presence of 3 drift (i.e., the simulated ocean tracers are not in balance with their bound4 ary conditions and the ocean circulation). Drift in simulated tracers adds

Email address: klindsay@ucar.edu (Keith Lindsay)

Preprint submitted to Ocean Modelling

November 1, 2016

C) 2016. This manuscript version is made available under the Elsevier user license http://www.elsevier.com/open-access/userlicense/1.0/ 
uncertainty to comparisons with observations and complicates the interpretation of tracer processes (e.g., source-sink terms and couplings between tracers), particularly when these processes are non-linear. Tracer drift also complicates the evaluation of tracer's response to transient forcing (e.g., rising atmospheric $\mathrm{CO}_{2}$ or anthropogenic nutrient inputs), because the tracer's response can depend on the state of the tracer itself. Therefore, the capacity to generate tracer distributions in balance with their boundary conditions and the ocean circulation is advantageous. Because ventilation of the deep ocean takes in excess of 1000 years (Holzer and Primeau, 2010; DeVries and Primeau, 2011; Khatiwala et al., 2012), brute force tracer spinup, running the tracers forward in time until this balance is reached, takes thousands of simulated years (i.e., multiple ventilation timescales). Running ocean models at a resolution typical of contemporary climate models (about $1^{\circ}$ or $\mathcal{O}\left(10^{6}\right)$ grid points) for this duration is computationally expensive, and often prohibitive.

Previous efforts have overcome this hurdle, in the context of offline tracer transport, by using Newton-Krylov based solvers to directly solve for the spun-up tracer distributions (Li and Primeau, 2008; Khatiwala, 2008; Bardin et al., 2014). One approach to spin up tracers for use in an online ocean model is to use a Newton-Krylov solver in conjunction with an offline tracer transport model, where the offline model is using the circulation of the online model. A disadvantage of this approach is that offline models typically use approximations in their representation of the online model's circulation (e.g., using temporally averaged circulation operators), and it is unclear how these approximations affect the spun-up tracer distributions. While Bardin et al. 
(2016) shows that the impact of these approximations on spun-up ideal age is small if the circulation is sampled frequently enough, the significance of the impact likely depends on the timescales of the processes affecting the tracers being spun up. Another disadvantage of this approach is that in practice, the tracers are either implemented separately in both the offline and online models, or the tracers are implemented in a way that is compatible with both models. For complex tracers, such as ecosystem models, the burden of having multiple tracer implementations, or of implementing the tracers compatibly with both models, can be excessive. Furthermore, it would not be easy to extend this approach to spinning up the active tracers of an ocean model. Because of these disadvantages, it is desirable to have a fast spin-up technique that is directly applicable to online ocean tracer simulations.

We present here the successful application of a Newton-Krylov based solver to efficiently spin up passive tracers in online ocean tracer simulations. We demonstrate this tool by efficiently spinning up the tracer ideal age (Thiele and Sarmiento, 1990) with respect to the circulation from different time intervals in a long physics run. We further evaluate how the spun-up ideal age tracer depends on the duration of the physics run, i.e., on how equilibrated the circulation is.

We describe, in Section 2, our formulation of the spin-up problem and the Newton-Krylov solver that we have developed to solve it, deferring some of the technical implementation details into appendices. We also describe in that section the computational experiments in which we use the solver. We present the results of these experiments in Section 3, discuss these results in Section 4, and give some concluding remarks in Section 5 . 


\section{Methods}

We first state the definition that we use of the spin-up problem for generic ocean tracers, mostly following the notation of Khatiwala (2008). Tracer concentrations, denoted by $\boldsymbol{c}(t)$, evolve in time according to the equation

$$
\frac{\mathrm{d} \boldsymbol{c}}{\mathrm{d} t}=\boldsymbol{f}(t, \boldsymbol{c})
$$

where $\boldsymbol{f}$ denotes the sum of mixing, advective, and source-sink tendencies of the tracers. In practice, $\boldsymbol{c}$ is a vector whose component values correspond to the ocean model's spatial discretization of tracers (e.g., grid cell means or spectral element decomposition coefficients). We use bold to denote vectors, such as $\boldsymbol{c}$, matrices, and other vector-valued operators. The solution of Eq. (1), with tracer concentrations at time $t_{0}$ specified by $\boldsymbol{c}\left(t_{0}\right)$, can be written as

$$
\boldsymbol{c}(t)=\boldsymbol{\Phi}\left(t, t_{0}, \boldsymbol{c}\left(t_{0}\right)\right)
$$

The spin-up problem is that for a given $t_{0}$ and time duration $\Delta t$, we seek initial tracer concentrations $\boldsymbol{c}\left(t_{0}\right)$ that leads to $\boldsymbol{c}\left(t_{0}+\Delta t\right)=\boldsymbol{c}\left(t_{0}\right)$. That is, we seek a solution $\boldsymbol{c}\left(t_{0}\right)$ of the equation

$$
\boldsymbol{G}\left(\boldsymbol{c}\left(t_{0}\right)\right) \equiv \boldsymbol{\Phi}\left(t_{0}+\Delta t, t_{0}, \boldsymbol{c}\left(t_{0}\right)\right)-\boldsymbol{c}\left(t_{0}\right)=\mathbf{0} .
$$

Such a solution is said to be spun-up with respect to the ocean model's circulation over the time interval $t_{0}$ to $t_{0}+\Delta t . \Delta t$ is typically an integer number of years. We refer the reader to Section 4.1 for some practical implications of this formal definition of the tracer spin-up problem.

The brute force approach to generating a spun-up solution to Eq. (3) is to run the tracers forward in time repeatedly from $t_{0}$ to $t_{0}+\Delta t$, initializing 
and the next Newton iterate is set to

$$
\boldsymbol{c}_{n+1}=\boldsymbol{c}_{n}+\delta \boldsymbol{c}_{n}
$$

91 Eq. (3),

$$
\boldsymbol{c}_{n+1}=\boldsymbol{\Phi}\left(t_{0}+\Delta t, t_{0}, \boldsymbol{c}_{n}\right) .
$$
expensive. the neighborhood of $\boldsymbol{c}_{n}$,

$$
\boldsymbol{J}_{n} \delta \boldsymbol{c}_{n}=-\boldsymbol{G}\left(\boldsymbol{c}_{n}\right)
$$

the tracer concentrations at $t_{0}$ from the previous values at $t_{0}+\Delta t$. Mathematically, this is equivalent to using fixed-point iterations on Eq. (2) to solve

We note that repeatedly performing fixed-point iterations is equivalent to running the tracers forward in time from $t_{0}$ to $t=\infty$, if one repeats periodically the model's circulation and physical state variables forward in time from the interval $t_{0}$ to $t_{0}+\Delta t$. As stated in Section 1, it takes thousands of model years to spin up tracers with such an approach, which is computationally

A different approach to find spun-up tracer concentrations is to apply Newton's method to Eq. (3). Newton's method is an iterative method to approximate the solution of a system of equations that proceeds as follows. Given the current iterate, $\boldsymbol{c}_{n}, \boldsymbol{G}(\boldsymbol{c})$ is approximated with a linearization in

$$
\boldsymbol{G}\left(\boldsymbol{c}_{n}+\delta \boldsymbol{c}_{n}\right) \approx \boldsymbol{G}\left(\boldsymbol{c}_{n}\right)+\boldsymbol{J}_{n} \delta \boldsymbol{c}_{n}
$$

$\delta \boldsymbol{c}_{n}$ is found such that the right-hand side of Eq. (5) is zero,

In these equations, $\boldsymbol{J}_{n}$ is the Jacobian of $\boldsymbol{G}$ evaluated at $\boldsymbol{c}_{n}$,

$$
\left.\boldsymbol{J}_{n} \equiv \frac{\partial \boldsymbol{G}}{\partial \boldsymbol{c}}\right|_{\boldsymbol{c}_{n}} .
$$


It represents how increments, $\delta \boldsymbol{c}$, to the tracer concentrations, $\boldsymbol{c}$, lead to changes in $\boldsymbol{G}$, as they evolve over the time interval $t_{0}$ to $t_{0}+\Delta t$. Because the duration of the interval, $\Delta t$, spans many model time steps (i.e., we are not considering instantaneous changes), $\boldsymbol{J}_{n}$ is not sparse, making it difficult to compute all of its entries. This precludes using a direct method to solve Eq. (6). We instead use an iterative method.

Krylov subspace methods (Saad, 2003) are a class of iterative methods for solving $\boldsymbol{A x}=\boldsymbol{b}$ that work by minimizing the residual $\boldsymbol{r}=\boldsymbol{b}-\boldsymbol{A x}$ over vectors from a Krylov subspace

$$
\mathcal{K}_{m}(\boldsymbol{A}, \boldsymbol{b})=\operatorname{span}\left\{\boldsymbol{b}, \boldsymbol{A} \boldsymbol{b}, \boldsymbol{A}^{2} \boldsymbol{b}, \ldots, \boldsymbol{A}^{m-1} \boldsymbol{b}\right\} .
$$

A notable feature of these methods is that they only utilize the matrix $\boldsymbol{A}$ through matrix-vector multiplications with $\boldsymbol{A}$, they do not directly access the individual entries of $\boldsymbol{A}$. Because of this feature, they are well suited for solving $\boldsymbol{A} \boldsymbol{x}=\boldsymbol{b}$ when the entries of $\boldsymbol{A}$ are not available, provided that one can compute matrix-vector multiplications with $\boldsymbol{A}$. Matrix-vector multiplications with our Jacobian matrix $\boldsymbol{J}_{n}$ can be approximated with the finite difference approximation

$$
\boldsymbol{J}_{n} \delta \boldsymbol{c} \approx\left(\boldsymbol{G}\left(\boldsymbol{c}_{n}+\sigma \delta \boldsymbol{c}\right)-\boldsymbol{G}\left(\boldsymbol{c}_{n}\right)\right) / \sigma,
$$

for a suitably chosen scalar $\sigma$. For our $\boldsymbol{G}$, the right-hand side of Eq. (10) can be evaluated with two model runs, one to evaluate each $\boldsymbol{G}$ term, or a single model run if $\boldsymbol{G}\left(\boldsymbol{c}_{n}\right)$ has been previously computed. Krylov subspace methods are thus a feasible way to solve Eq. (6). The particular Krylov subspace method that we use is GMRES (Saad and Schultz, 1986), following the presentation in Saad (2003). 
Summarizing, we use Newton's method, Eqs. (6) and (7), to solve Eq. (3). The increment in Newton's method, $\delta \boldsymbol{c}_{n}$, is obtained by using GMRES to solve Eq. (6) . The matrix-vector multiplications required by GMRES are evaluated using Eq. (10). There are a number of technical details involved in implementing this Newton-Krylov solver. In particular, the batch computing environment that our ocean model is run within leads to some complications. We refer the reader to Appendix A for these technical details.

\subsection{Krylov Preconditioner}

Following Li and Primeau (2008) and Khatiwala (2008), we apply a preconditioner to Eq. (6) to improve the convergence rate of the Krylov solver. We use a left preconditioner, yielding

$$
\boldsymbol{P}_{n} \boldsymbol{J}_{n} \delta \boldsymbol{c}_{n}=-\boldsymbol{P}_{n} \boldsymbol{G}\left(\boldsymbol{c}_{n}\right)
$$

where $\boldsymbol{P}_{n}$ is the preconditioner matrix. In order to improve the convergence rate of the Krylov solver, $\boldsymbol{P}_{n}$ should approximate the inverse of $\boldsymbol{J}_{n}$. With the introduction of a left preconditioner into the Krylov solver, matrix operations using the matrix $\boldsymbol{J}_{n}$, such as matrix-vector multiplications, are replaced with matrix operations using the matrix $\boldsymbol{P}_{n} \boldsymbol{J}_{n}$. These latter matrix-vector multiplications are implemented by first evaluating a matrix-vector multiplication using $\boldsymbol{J}_{n}$, evaluated using Eq. (10), and then multiplying the result by $\boldsymbol{P}_{n}$. In order to be of practical benefit, computing matrix-vector multiplications with $\boldsymbol{P}_{n}$ needs to be computationally feasible.

We use the same type of conditioner that $\mathrm{Li}$ and Primeau (2008) and Khatiwala (2008) both use. Following the derivation in Khatiwala (2008), we construct $\boldsymbol{P}_{n}$ by introducing $\tilde{\boldsymbol{\Phi}}$, an approximation to the ocean model's 
evaluation of $\boldsymbol{\Phi}$, and set $\boldsymbol{P}_{n}$ to be inverse of $\tilde{\boldsymbol{J}}$, the Jacobian that corresponds to $\tilde{\boldsymbol{\Phi}}$. As shown by Khatiwala (2008), a choice for $\tilde{\boldsymbol{\Phi}}$ that works well is to replace the ocean model's time stepping with a single backward Euler time step over the time interval $t_{0}$ to $t_{0}+\Delta t$,

$$
\tilde{\boldsymbol{\Phi}}_{K}-\boldsymbol{c}\left(t_{0}\right)=\Delta t \boldsymbol{f}\left(t_{0}+\Delta t, \tilde{\boldsymbol{\Phi}}_{K}\right)
$$

where the $K$ subscript denotes that this is the approximation of Khatiwala (2008). In order to better capture the mean of the ocean model's circulation, we replace the instantaneous mixing and advective operators that effectively appear on the right-hand side of Eq. (12) with their time-average over the time interval $t_{0}$ to $t_{0}+\Delta t$, yielding

$$
\tilde{\boldsymbol{\Phi}}-\boldsymbol{c}\left(t_{0}\right)=\Delta t \overline{\boldsymbol{f}}(\tilde{\boldsymbol{\Phi}})
$$

where the overbar represents time-averaging. Note that while the operators of $\overline{\boldsymbol{f}}$ are time-averaged, they are being applied in Eq. (13) to instantaneous values of $\tilde{\boldsymbol{\Phi}}$. One can derive, see Appendix B, that the inverse of $\tilde{\boldsymbol{J}}$ for this $\tilde{\boldsymbol{\Phi}}$ is

$$
\boldsymbol{P}_{n}=\tilde{\boldsymbol{J}}^{-1}=\left(\Delta t \frac{\partial \overline{\boldsymbol{f}}}{\partial \boldsymbol{c}}\right)^{-1}-\boldsymbol{I},
$$

where $\boldsymbol{I}$ is the identity matrix. It then follows that the matrix-vector product $\boldsymbol{P}_{n} \boldsymbol{v}$, for an arbitrary vector $\boldsymbol{v}$, is

$$
\boldsymbol{P}_{n} \boldsymbol{v}=\left(\Delta t \frac{\partial \overline{\boldsymbol{f}}}{\partial \boldsymbol{c}}\right)^{-1} \boldsymbol{v}-\boldsymbol{v}
$$

52 Computing this requires solving

$$
\left(\Delta t \frac{\partial \overline{\boldsymbol{f}}}{\partial \boldsymbol{c}}\right) \boldsymbol{x}=\boldsymbol{v}
$$


for $\boldsymbol{x}$. The matrix that appears in this system of linear equations, $\Delta t \partial \overline{\boldsymbol{f}} / \partial \boldsymbol{c}$, represents how instantaneous tracer tendencies from the time-averaged operators of $\overline{\boldsymbol{f}}$ depend on tracer concentrations. These partial derivatives, of $\overline{\boldsymbol{f}}$ with respect to $\boldsymbol{c}$, are localized in space, because they are of instantaneous, not time-integrated, quantities. This localization in turn leads to $\Delta t \partial \overline{\boldsymbol{f}} / \partial \boldsymbol{c}$ being sparse. Systems of linear equations with sparse matrices, such as this, can be solved with existing sparse matrix libraries, such as SuperLU (Li et al., 1999; Li and Demmel, 2003). Because some aspects of the construction of this matrix are specific to our ocean model, we defer a description of the construction of this matrix to Section 2.3. after we have described our ocean model.

\subsection{Ocean Model Description}

We have implemented the Newton-Krylov solver described above in the ocean component (Danabasoglu et al., 2012) of version 1 of the Community Earth System Model (CESM1) (Hurrell et al., 2013), which is a based on version 2 of the Parallel Ocean Program (POP2) (Smith et al., 2010). In our numerical experiments, POP2 uses a nominal $1^{\circ}$ curvilinear grid, with 320 grid cells in the zonal direction and 384 grid cells in the meridional direction. The vertical grid has 60 layers. At this spatial resolution, a parameterization of the effects of mesoscale eddies is necessary, and POP2 includes the GentMcWilliams isopycnal mixing scheme (Gent and McWilliams, 1990). As described in Danabasoglu et al. (2012), some of the other parameterizations of POP2 include the KPP vertical mixing scheme (Large et al., 1994) and a parameterization of overflows (Danabasoglu et al., 2010). Our experiments use an active sea ice model, the sea ice component of CESM1 (Holland et al., 
2012), which is based on version 4 of the Los Alamos Sea Ice Model (CICE4) (Hunke and Lipscomb, 2008). The sea ice model uses the same horizontal grid as the ocean model. The surface forcing in our experiments is the normal year (NY) forcing of Large and Yeager (2004).

\subsection{Applying the Krylov Preconditioner}

As stated in Section 2.1, computing the matrix-vector product $\boldsymbol{P}_{n} \boldsymbol{v}$ involves solving the system of linear equations in Eq. (16) and applying Eq. (15). So it is necessary to construct the matrix $\Delta t \partial \overline{\boldsymbol{f}} / \partial \boldsymbol{c}$, subsequently denoted by $\boldsymbol{Q}$, and solve the corresponding system of linear equations. The entries of the matrix $\boldsymbol{Q}$ are the partial derivatives of the tracer tendencies from the time-averaged mixing, advective, and source-sink operators with respect to the tracer concentrations.

Motivated by the transport matrix method of Khatiwala et al. (2005), computation of the partial derivatives of tendencies from time-averaged linear operators (e.g., lateral mixing and advection) can be done as follows. We write tracer concentrations as a weighted sum of Kronecker delta functions

$$
\boldsymbol{c}=\sum_{i} c^{i} \boldsymbol{\delta}^{i}
$$

where $i$ is a grid cell index, $c^{i}$ is the value of $\boldsymbol{c}$ at grid cell $i$, and $\boldsymbol{\delta}^{i}$ is a Kronecker delta function at grid cell $i$ (i.e., $\boldsymbol{\delta}^{i}$ is one at grid cell $i$ and zero at other grid cells). Denoting the linear operator by $\mathcal{L}$, and its time-average by $\overline{\mathcal{L}}$, we have

$$
\overline{\mathcal{L}}(\boldsymbol{c})=\overline{\mathcal{L}}\left(\sum_{i} c^{i} \boldsymbol{\delta}^{i}\right)=\sum_{i} c^{i} \overline{\mathcal{L}}\left(\boldsymbol{\delta}^{i}\right)
$$


where we have used the linearity of $\overline{\mathcal{L}}$. The derivative of $\overline{\mathcal{L}}(\boldsymbol{c})$ with respect to $c^{j}$ is thus

$$
\frac{\partial \overline{\mathcal{L}}(\boldsymbol{c})}{\partial c^{j}}=\sum_{i} \frac{\partial c^{i}}{\partial c^{j}} \overline{\mathcal{L}}\left(\boldsymbol{\delta}^{i}\right)=\overline{\mathcal{L}}\left(\boldsymbol{\delta}^{j}\right)=\overline{\mathcal{L}\left(\boldsymbol{\delta}^{j}\right)}
$$

where the final equality holds because $\boldsymbol{\delta}^{j}$ is independent of $t$. This shows that the partial derivatives of the tendencies from $\overline{\mathcal{L}}$ are equal to the time-averaged tendencies of $\mathcal{L}$ applied to Kronecker delta functions.

We compute these terms with the aid of impulse response (IR) tracers, which are tracers that are reset every time step to Kronecker delta functions (i.e., impulses). The desired partial derivatives are the time-average of the result of applying $\mathcal{L}$ to these IR tracers, which can be included in the ocean model's diagnostic output. A naïve implementation of the IR tracers would have one tracer per grid cell. This would lead to $\mathcal{O}\left(10^{6}\right)$ tracers for our model grid, which is not practical to run with. However, for operators that are localized in space, one can superimpose IR tracers for grid cells that are separated spatially and recover the tendencies of the non-superimposed IR tracers from the tendencies of the superimposed IR tracers. That is, the tendencies $\overline{\mathcal{L}\left(\boldsymbol{\delta}^{i}\right)}$ and $\overline{\mathcal{L}\left(\boldsymbol{\delta}^{j}\right)}$ can be recovered from $\overline{\mathcal{L}\left(\boldsymbol{\delta}^{i}+\boldsymbol{\delta}^{j}\right)}$ if $i$ and $j$ are sufficiently separated spatially. This principle can be used to superimpose IR tracers for many grid cells into a single tracer, so that the $\overline{\mathcal{L}\left(\boldsymbol{\delta}^{j}\right)}$ terms can be computed with fewer tracers than would be used in the naïve implementation. We use this approach to compute the $\overline{\mathcal{L}\left(\boldsymbol{\delta}^{j}\right)}$ terms for the lateral mixing terms of $\boldsymbol{Q}$. The locality of this operator allows these terms to be computed with $\approx 30$ IR tracers.

The IR tracer approach described above can also be used to compute the $\overline{\mathcal{L}\left(\boldsymbol{\delta}^{j}\right)}$ terms of $\boldsymbol{Q}$ for the advective operator. However, this would require 
more IR tracers, because the advective operator in our ocean model has a wider computational stencil than the lateral mixing operator. So we have opted to use a less computationally demanding, though model specific, approach for the advective terms of $\boldsymbol{Q}$. Because the advective operator, denoted by $\mathcal{A}$, is linear with respect to tracer concentrations, we have

$$
\frac{\partial \overline{\mathcal{A}}(\boldsymbol{u}, \boldsymbol{c})}{\partial c^{j}}=\overline{\mathcal{A}\left(\boldsymbol{u}, \boldsymbol{\delta}^{j}\right)},
$$

where $\boldsymbol{u}$ denotes the (time-varying) advecting velocity. However, because the tracer advection scheme used in our ocean model is upwind biased (Holland et al., 1998), the time-averaged advective operator is not equal to the advective operator obtained using time-averaged velocities. However, the time-averaged advective operator is equal to the sum of the advective operators obtained using the time-average of the positive and negative parts of the velocity. That is,

$$
\begin{aligned}
\overline{\mathcal{A}\left(\boldsymbol{u}, \boldsymbol{\delta}^{j}\right)} & =\overline{\mathcal{A}\left(\boldsymbol{u}^{+}, \boldsymbol{\delta}^{j}\right)+\mathcal{A}\left(\boldsymbol{u}^{-}, \boldsymbol{\delta}^{j}\right)} \\
& =\mathcal{A}\left(\overline{\boldsymbol{u}^{+}}, \boldsymbol{\delta}^{j}\right)+\mathcal{A}\left(\overline{\boldsymbol{u}^{-}}, \boldsymbol{\delta}^{j}\right) .
\end{aligned}
$$

Combining these equations, we have

$$
\frac{\partial \overline{\mathcal{A}}(\boldsymbol{u}, \boldsymbol{c})}{\partial c^{j}}=\mathcal{A}\left(\overline{\boldsymbol{u}^{+}}, \boldsymbol{\delta}^{j}\right)+\mathcal{A}\left(\overline{\boldsymbol{u}^{-}}, \boldsymbol{\delta}^{j}\right) .
$$

We use this equality to compute the advective terms of $\boldsymbol{Q}$. We compute $\overline{\boldsymbol{u}^{+}}$and $\overline{\boldsymbol{u}^{-}}$online, and evaluate $\mathcal{A}\left(\overline{\boldsymbol{u}^{+}}, \boldsymbol{\delta}^{j}\right)$ and $\mathcal{A}\left(\overline{\boldsymbol{u}^{-}}, \boldsymbol{\delta}^{j}\right)$ offline, using an implementation of the model's $\mathcal{A}$ in the offline code that constructs $\boldsymbol{Q}$.

Vertical mixing in our ocean model is implemented implicitly in time, so the computational stencil for this operator spans the entire water column. 
We do not use the IR tracer approach for these terms of $\boldsymbol{Q}$ because of this, as it would require many more IR tracers. Instead, we compute the timeaverage of the vertical mixing coefficients online and use these, along with an implementation of the model's vertical difference operator, in the offline code that constructs $\boldsymbol{Q}$.

As described in Danabasoglu et al. (2012), our ocean model includes an overflow parameterization. In this parameterization, tracer values in an overflow's source region instantaneously affect tracer values in the corresponding entrainment and outflow regions. This non-local dependence between tracer values from spatially separated grid cells increases the band-width of the otherwise sparse $\boldsymbol{Q}$, which increases the fill-in when the matrix is factored for solving (see below). We have omitted these terms from $\boldsymbol{Q}$ and still achieved satisfactory rates of convergence.

The final terms needed in $\boldsymbol{Q}$ are the partial derivatives of the tracer source-sink tendencies with respect to the tracer concentrations. Because these tendencies do not in general depend on the tracer concentrations in a linear way, the IR tracer approach described above is not applicable. For tracers with simple source-sink terms, these derivatives can be computed directly with analytical derivatives of the source-sink terms. More generally, the derivatives can be approximated with finite difference approximations (i.e., evaluate the source-sink tendencies with perturbed and unperturbed tracer values and apply standard finite difference formulas). Some sourcesink tendencies, like the setting of ideal age to zero in the surface layer, are not differentiable with respect to the tracer concentrations. For the derivatives of these terms, we replace the tendency with a differentiable approximation 
and include the derivative of the replacement in $\boldsymbol{Q}$. We emphasize that this approximation is only used in the preconditioner for the Krylov solver, through its usage in $\boldsymbol{Q}$. The source-sink tendency in the online model is unchanged. For ideal age, we approximate the setting of ideal age to zero with a restoring to zero term, using a timescale of 1 hour.

Most of the terms of $\boldsymbol{Q}$ described above, such as those computed with the IR tracer approach and those that arise from linear source-sink terms, are independent of the tracer concentrations. Because of this, these terms can be computed once, for a given time interval $t_{0}$ to $t_{0}+\Delta t$, and used repeatedly for all Newton and Krylov iterations. Non-linear tracer source-sink tendencies however, lead to terms in $\boldsymbol{Q}$ that depend on tracer concentrations. These terms need to be recomputed for each Newton iteration, as the tracer state changes from one Newton iteration to the next.

Once $\boldsymbol{Q}$ is constructed, computing the matrix-vector product $\boldsymbol{P}_{n} \boldsymbol{v}$ involves solving the system of linear equations in Eq. (16) and applying Eq. (15). We refer the reader to Appendix $\mathrm{C}$ for the details on how we utilize the library SuperLU_DIST (Li et al., 1999; Li and Demmel, 2003) to solve Eq. (16).

\subsection{Experiment Description}

The Newton-Krylov solver described above spins up tracers with respect to the model's circulation over a time interval $t_{0}$ to $t_{0}+\Delta t$. In our experiments, we have applied the solver to spin up the tracer ideal age (Thiele and Sarmiento, 1990). The ideal age of a water parcel is defined as the duration of time since the parcel was last in contact with the sea surface. The tracer ideal age is set to zero in the surface layer at every model time

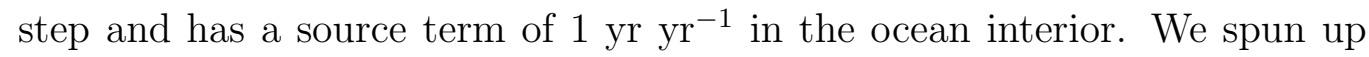


ideal age with respect to the model's circulation over one-year intervals at multiple times in a 1000-year long NY forced physics run. One year was chosen because at this model resolution, the one-year period of the NY forcing leads to circulation that is approximately periodic with the same period. Longer durations would be appropriate for circulation fields with interannual variability, whether it is externally forced or internally generated. The tracer spin-ups were performed at multiple points along the physics run in order to evaluate how the duration of the physics run, i.e., how equilibrated the circulation is, affects the corresponding spun-up tracer solution. The ocean model in the physics run was started at a state of rest; temperature and salinity were initialized from the January-mean of the Polar Science Center Hydrographic Climatology (PHC2), which is constructed using data from Levitus et al. (1998), blending in data from Steele et al. (2001) for the Arctic Ocean. The spin-ups of ideal age were performed at multiple points in time in the physics run, at 50-year intervals up to year 500 of the physics run, and 100-year intervals from there up to year 1000. We performed one-year runs at each of these times to generate diagnostic output necessary to apply the preconditioner for the Krylov solver (see Section 2.3).

\section{Results}

We now present results of the applying to Newton-Krylov solver, which is using Eq. (7) to find a solution to Eq. (3), to the experimental framework described above. 


\subsection{Convergence of Solver}

Our first test of the solver is to confirm that $\left\|\boldsymbol{G}\left(\boldsymbol{c}_{n}\right)\right\| \rightarrow 0$ as $n$ increases, where $\left\|\boldsymbol{G}\left(\boldsymbol{c}_{n}\right)\right\|$ is the global, volume weighted root-mean-square change in ideal age over the year of integration. We do this initial verification in two separate runs of the solver, using the circulation from years 250 and 500 of the physics run (Fig. 17a). In these solver runs, the first Newton iterate, $\boldsymbol{c}_{0}$, for ideal age was all zeros and the Krylov solver was hard-coded to use four iterations. After a few Newton iterations, $\left\|\boldsymbol{G}\left(\boldsymbol{c}_{n}\right)\right\|$ decreases exponentially with $n$, verifying convergence. The rate of convergence is similar for the different years of circulation. We note that in both of these runs of the solver, the Armijo adjustment (Appendix Ad to the Newton increment was invoked once for $n=0$, and was not necessary for subsequent Newton iterations.

Another metric of convergence is that the fraction of the ocean's volume where $\left|\boldsymbol{G}\left(\boldsymbol{c}_{n}\right)\right|$ exceeds a particular threshold should decrease to 0 as $n$ increases. For ideal age, we use a threshold of $10^{-2}$ yrs/yr (Fig. 1b). This threshold is comparable to the natural abiotic radiocarbon equilibrium criterion in the Ocean Carbon Model Intercomparison Project, phase 2 (OCMIP2) protocol, which stated that $98 \%$ of the ocean volume should have a drift less than $10^{-3}$ permil/yr (Orr et al., 2000). In our convergence test runs, the fraction of the ocean's volume where the change in ideal age exceeds our threshold decreases exponentially with $n$, verifying convergence. The fraction of the ocean's volume below our threshold drops below $2 \%$ after five Newton iterations.

Another way of examining this convergence is to visualize the volume weighted histogram of $\log _{10}\left|\boldsymbol{G}\left(\boldsymbol{c}_{n}\right)\right|$. We do this for two different values of 
$n$ in the convergence test runs using the circulation from year 250 of the physics run (Fig. 2). An advantage of these histograms over the depiction of global measures in Fig. 1 is the additional information on the distribution of $\log _{10}\left|\boldsymbol{G}\left(\boldsymbol{c}_{n}\right)\right|$ that is provided (e.g., mean, spread, and extent of the distribution's tails). The histograms indicate that $\left|\boldsymbol{G}\left(\boldsymbol{c}_{n}\right)\right|$ is approximately log-normally distributed. Consistent with convergence, the mean of $\log _{10}\left|\boldsymbol{G}\left(\boldsymbol{c}_{n}\right)\right|$ decreases with $n$. Additionally, the standard deviation increases with $n$, though at a much smaller rate than the decrease in the mean.

\subsection{Stability of Simulations Initialized with Spun-up Tracers}

As alluded to in Section 1, one of the motivations for spinning up ocean tracers is to initialize simulations. We evaluate the utility of the NewtonKrylov solver for this purpose with two 10-year long experiments, initializing ideal age with one of the spun-up states described above. The ocean model's physical state was initialized to the beginning of year 250 from the physics run. Ideal age was initialized to the state obtained with 5 Newton iterations using the circulation of year 250. In the first experiment, the ocean model's physical state was reset at the end of every year to its state from the beginning of year 250, causing the ocean's circulation to repeat the year 250 circulation 10 times. The purpose of this experiment is to evaluate how ideal age changes over multiple years, but with a circulation field identical to that used in the Newton-Krylov solver. The change in ideal age in this experiment (Fig. 3a) was comparable to its residuals after 5 Newton iterations (Fig. 2a). In the second experiment, the ocean model's physical state was allowed to freely evolve, yielding drift and internally generated variability in the ocean circulation. The purpose of this experiment is to evaluate how 

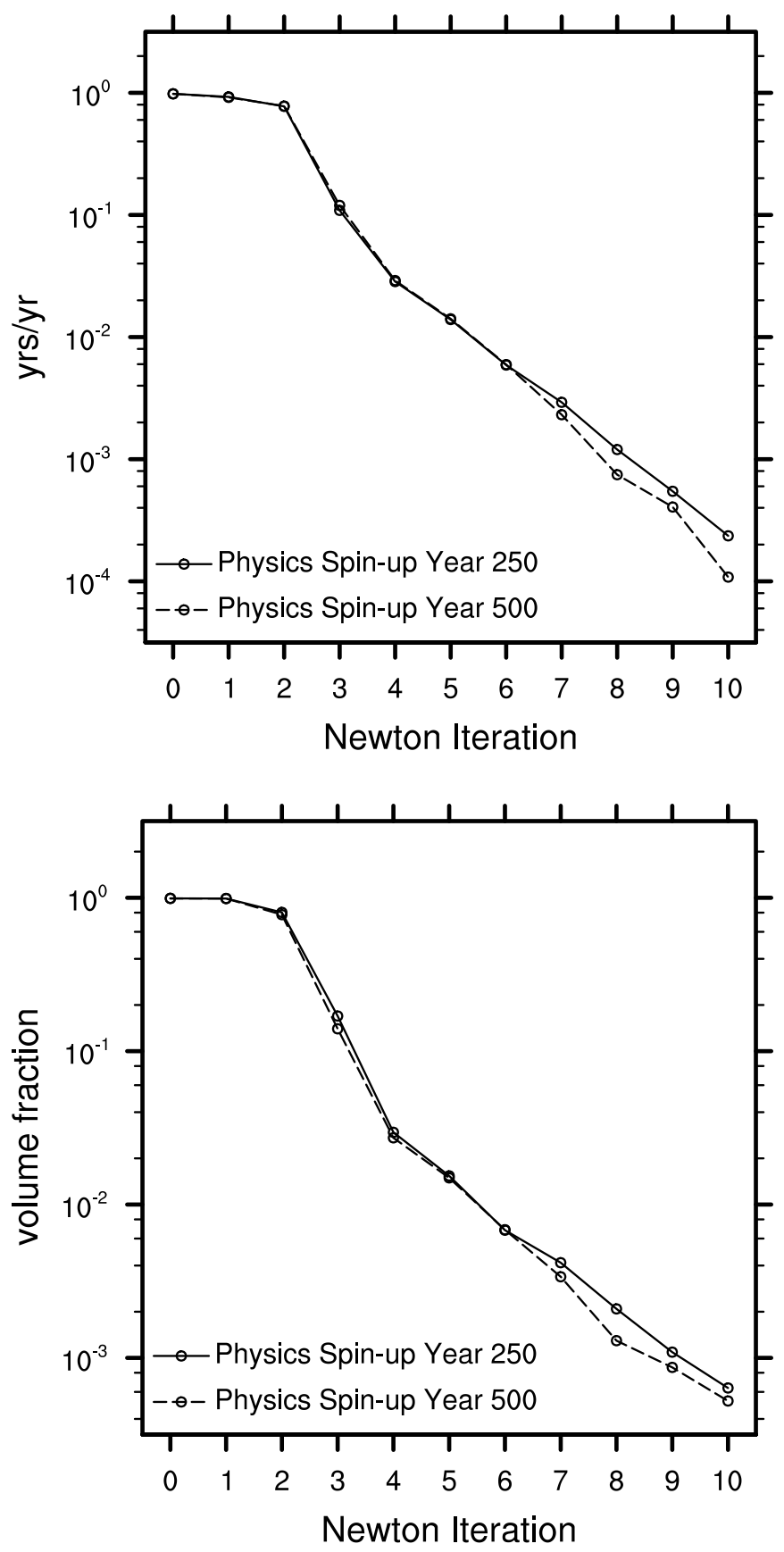

Figure 1: Convergence of the tracer ideal age across Newton iterates: (a) Volume weighted root-mean-square of $\boldsymbol{G}$ (see Eq. (3) ), (b) Fraction of ocean's volume where $\boldsymbol{G}$ exceeds the threshold $10^{-2}$ yrs/yr. The Armijo adjustment was applied once in the first Newton iteration. 

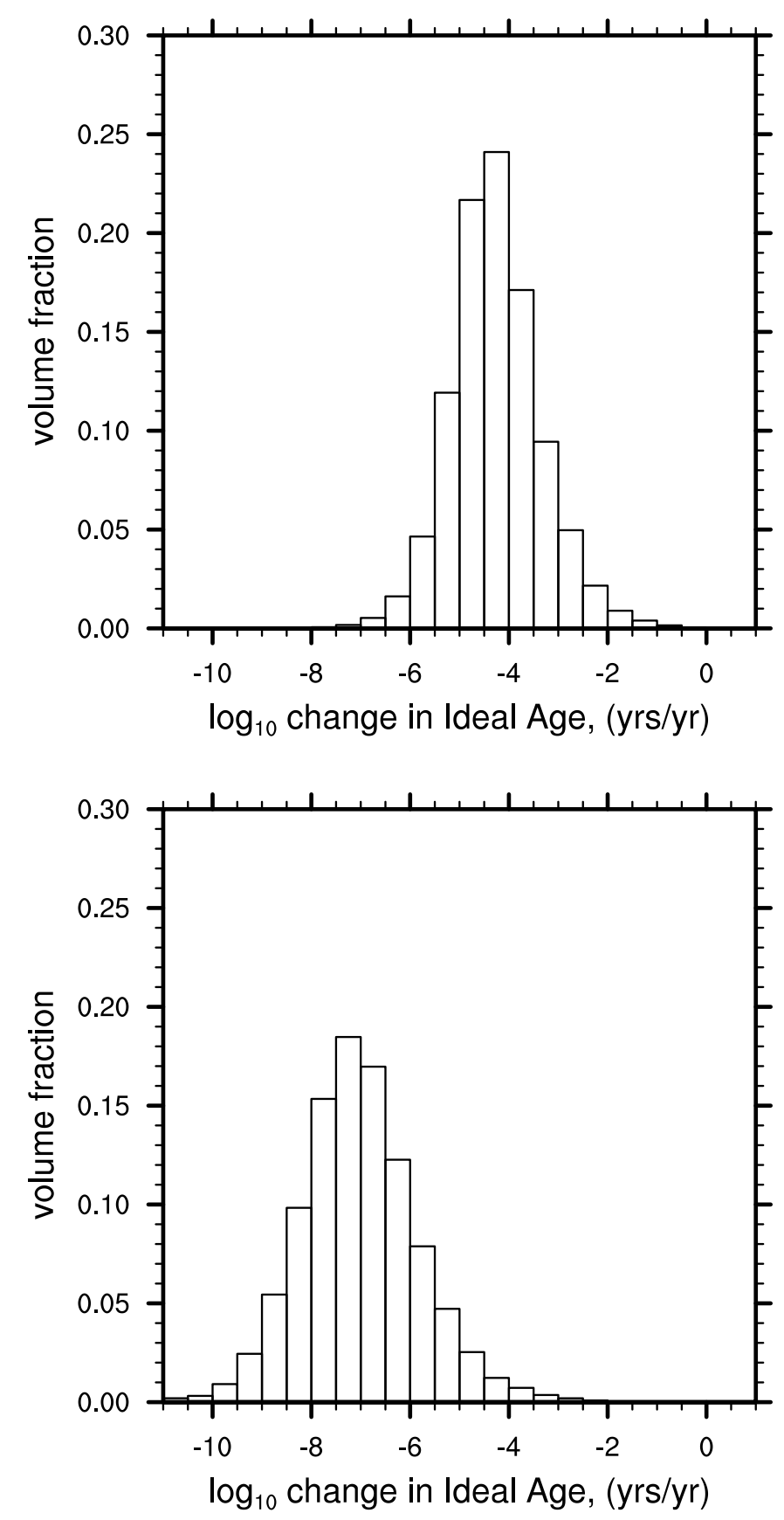

Figure 2: Volume weighted histogram of $\log _{10}\left|\boldsymbol{G}\left(\boldsymbol{c}_{n}\right)\right|$ (see Eq. (3)): (a) Newton iteration 5 , (b) Newton iteration 10. Ocean circulation was year 250 of the physics run. 
the change in ideal age is impacted by this change in the ocean circulation away from that used in the Newton-Krylov solver. The change in ideal age in this experiment (Fig. 3b) was considerably larger than the residuals after 5 Newton iterations: only $42 \%$ of the ocean's volume maintained change in ideal age below our threshold of $10^{-2}$ yrs/yr. However, the change was below a relaxed threshold of $5 \cdot 10^{-2} \mathrm{yrs} / \mathrm{yr}$ for $80 \%$ of the ocean's volume.

An analogous pair of experiments were run where ideal age was initialized to the state obtained with 10 Newton iterations. The volume weighted histograms, not shown, portray results similar to those above. In the experiment with year 250 circulation repeating, change in ideal age was comparable to the residuals after 10 Newton iterations, demonstrating that for repeating circulation, the more converged solution maintains its state. In the experiment with freely evolving circulation, change in ideal age was larger and very similar to the change in the freely evolving circulation experiment initialized after 5 Newton iterations, demonstrating that for freely evolving circulation, the 5 additional Newton iterations have limited benefit.

\subsection{Computational Performance}

In the convergence test runs described in Section 3.1, our convergence criterion was reached with 5 Newton iterations, using a tracer distribution of all zeros as the initial Newton iterate. Each Newton iteration used 4 Krylov iterations, resulting in $5 \cdot 5=25$ evaluations of $\boldsymbol{G}$ and the same number of inversions of the Krylov preconditioner. In our implementation, solving the system of linear equations related to the preconditioner takes about the same number of CPU hours as running the ocean model with ideal age for 1 year. So running the Newton-Krylov solver to this convergence criterion 

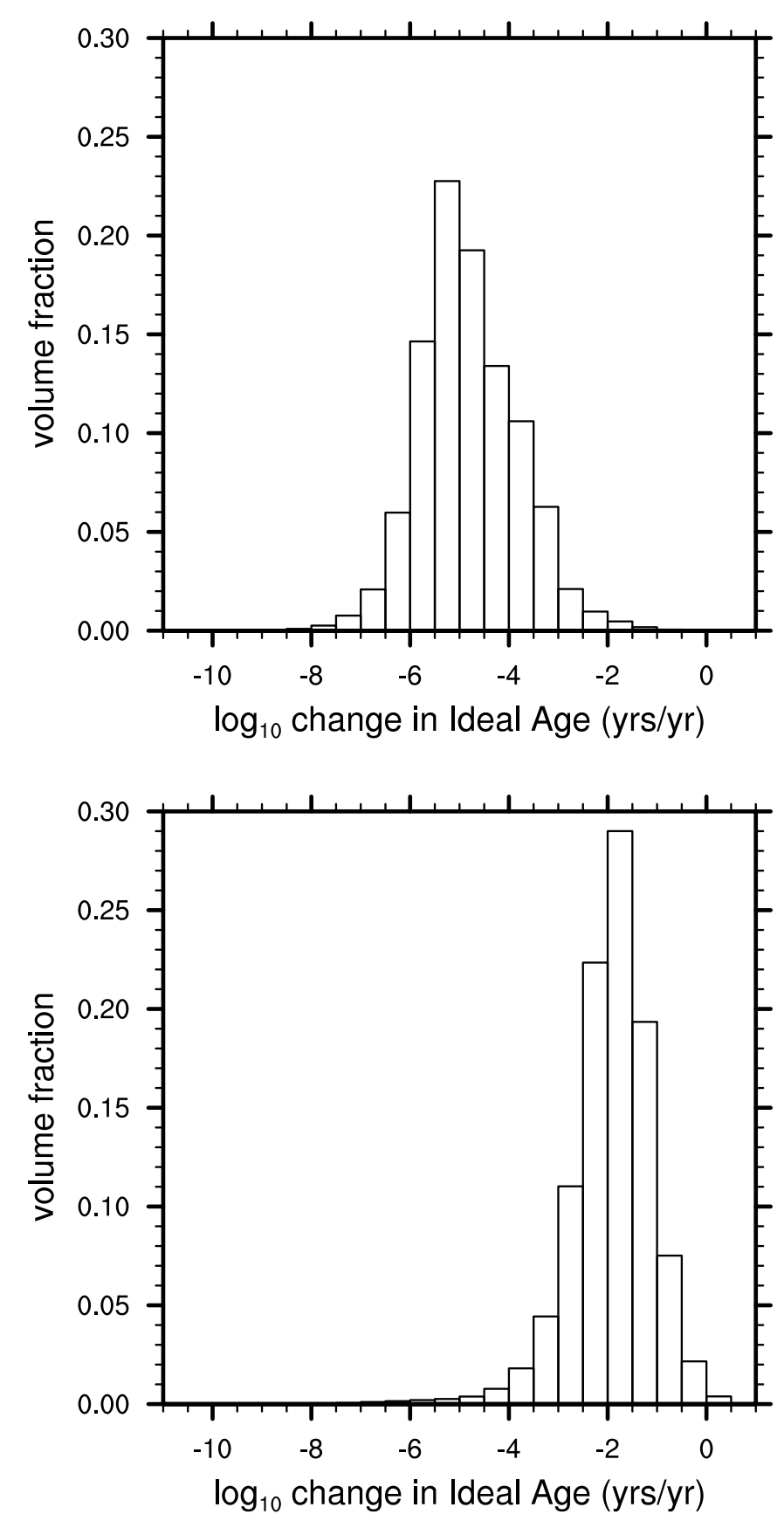

Figure 3: Volume weighted histogram of change in ideal age across a 10-year run in ideal age with spun-up ideal age used for initial state: (a) circulation repeats that used in ideal age spin-up, (b) circulation evolves freely over 10-year run. Ideal age was initialized from Newton iteration 5 . The ocean physical state was initialized from year 250 of the physics run. 
was computationally equivalent to running the ocean model with ideal age for 50 years. We project, using ideal age results from the physics run (not shown) that it would take over 10,000 years to reach this criterion using the brute force spin-up approach. So the Newton-Krylov solver is faster than the brute force spin-up approach by a factor over 200, even with an initial Newton iterate of all zeros. In practice, one would use a better initial Newton iterate. For instance, in our Newton-Krylov spin-ups using different years of circulation from the physics run (Section [3.4), we were able to satisfy the convergence criterion with 2 Newton iterations by using better initial Newton iterates, leading to the Newton-Krylov solver being faster than the brute force spin-up approach by a factor of over 500. In these applications of the solver, the initial ideal age iterate for a given year in the physics run was the spun-up ideal age from the preceding ideal age spin-up. This type of approach, initializing the spin-up with a previously spun-up solution, would be beneficial when evaluating the impact of a varying a model parameter on spun-up tracers.

\subsection{Dependence of Spun-up Tracer Concentrations on Duration of Physics Run}

As an application of the solver, we have spun up ideal age at multiple time intervals in the long physics run. The resulting spun-up ideal age distributions can be used to address questions that arise from different perspectives. One perspective is that of using ventilation tracers, like ideal age, as a tool to better understand the circulation of the ocean. From this perspective, the different spun-up ideal age distributions have the potential, through ideal age's integrative nature, to reveal aspects of the evolving model's circulation 
that would not be readily apparent from examining only the temperature, salinity, and velocity fields of the model. An example of a question that arises from this perspective is: How is the ventilation of the ocean varying as the density field of the model adjusts on long timescales? Another perspective is that of using the solver to spin up tracers for the purpose of using or understanding the spun-up tracers themselves, not necessarily as a tool to understand the ocean's circulation. An example of a question that arises from this perspective is: How long does physics run need to be in order for the corresponding spun-up tracers to be robust? In the following, we are focusing on this latter perspective.

The circulation in the long physics run has drift that persists for the duration of the run. As the circulation drifts, the corresponding spun-up ideal age values coevolve. In the following, we refer to this evolution of the spun-up ideal age values as drift. We now describe how these spun-up ideal age values, and their drift, depend on the duration of the physics run, i.e., how equilibrated the circulation is. We first examine zonal means of ideal age at $35^{\circ} \mathrm{N}$ in the Pacific and Atlantic basins as a function of the year in the physics run to which ideal age is spun up with respect to (Fig. 4). After the physics run has been run for a sufficiently long duration, the corresponding spun-up ideal age stabilizes, and becomes essentially constant as the physics run is extended. In the Pacific zonal mean, this occurs for ideal age in the top $750 \mathrm{~m}$ after a few hundred years. For instance, in the top $750 \mathrm{~m}$, ideal age is within $10 \%$ of its year 1000 values by year 450 . In the older waters of the deep Pacific, the drift in the spun-up ideal age values persists for the full duration of the physics run. At year 1000 of the physics run, the derivative 
of the spun-up ideal age with respect to the physics run year exceeds 0.25 yrs/yr at all depths deeper than $2500 \mathrm{~m}$. Spun-up ideal age at $35^{\circ} \mathrm{N}$ in the Atlantic stabilizes more quickly than in the Pacific; it is within $10 \%$ of its year 1000 values by year 600 at all depths. Its drift is below the threshold of $5 \cdot 10^{-2} \mathrm{yrs} / \mathrm{yr}$ at all depths shallower than $2000 \mathrm{~m}$ by year 450 and all depths shallower than $3000 \mathrm{~m}$ by year 700 .

As mentioned above, a question that arises from the perspective that we are focusing on is: How long does physics run need to be in order for the corresponding spun-up tracers to be robust? We quantify this for zonal means of ideal age in the Pacific and Atlantic basins by computing, for each grid point, the time after which the relative drift in spun-up ideal age is below the threshold of $2 \cdot 10^{-4} \mathrm{yrs} / \mathrm{yr}^{2}$ (Fig. 林). Relative drift is the derivative of the spun-up ideal age with respect to the physics run year divided by the spunup ideal age. We use this relative drift criterion here because it seems to us to be of more practical use for ideal age than the OCMIP2 related absolute drift criterion. In the Pacific zonal mean, this threshold is reached by year 900 of the physics run for much of the basin. Shallower than $4000 \mathrm{~m}$, this threshold is reached in most of the southern hemisphere by year 500. In the Atlantic zonal mean, this threshold is reached by year 800 of the physics run for much of the basin south of $60^{\circ} \mathrm{N}$. Shallower than $2000 \mathrm{~m}$, this threshold is reached in most of the southern hemisphere by year 500 .

It is likely that the duration required for the physics run depends on the physical parameterizations of the ocean model and the model's state at the beginning of the physics run, so the timescales described above should not be considered universal, even for a specific tracer, like ideal age. An alter- 
native approach to determining how long the physics run needs to be is to functionally relate spun-up tracer values to diagnostics of the ocean model's circulation. One could then run the physics run until these circulation diagnostics have small drift. It is fair to hope that such relationships would be applicable to ocean models with different physical parameterizations. While determining such relationships is not straightforward, intuition about the ocean's circulation can suggest relationships to examine. For example, it is reasonable to expect that ideal age in the deep north Pacific is inversely related to volumetric transport into the deep Pacific from the south. Indeed, in our experiments, spun-up ideal age averaged across the Pacific at $3500 \mathrm{~m}$ and $45^{\circ} \mathrm{N}$ is inversely proportional to the modeled transport integrated from $3500 \mathrm{~m}$ to $5500 \mathrm{~m}$ across the Pacific at $35^{\circ} \mathrm{S}$ (Fig. 6).

\section{Discussion}

\subsection{Tracer Spin-up Definition}

In Section 2, we introduced Eq. (3) as our definition of the tracer spin-up problem. This definition is intuitive when the ocean model's circulation is periodic with period $\Delta t$. However, it is not clear how appropriate this definition is when the circulation is not periodic (e.g., when the surface forcing has interannual variability or the ocean model has high enough spatial resolution to support internally generated variability). In our experience with using the Newton-Krylov solver in the presence of interannually varying circulation, not shown here, the Newton-Krylov solver does converge to a solution of Eq. (3). However, this solution is only useful (e.g., for initializing runs) if the circulation over the time interval $t_{0}$ to $t_{0}+\Delta t$ is representative of the circula- 

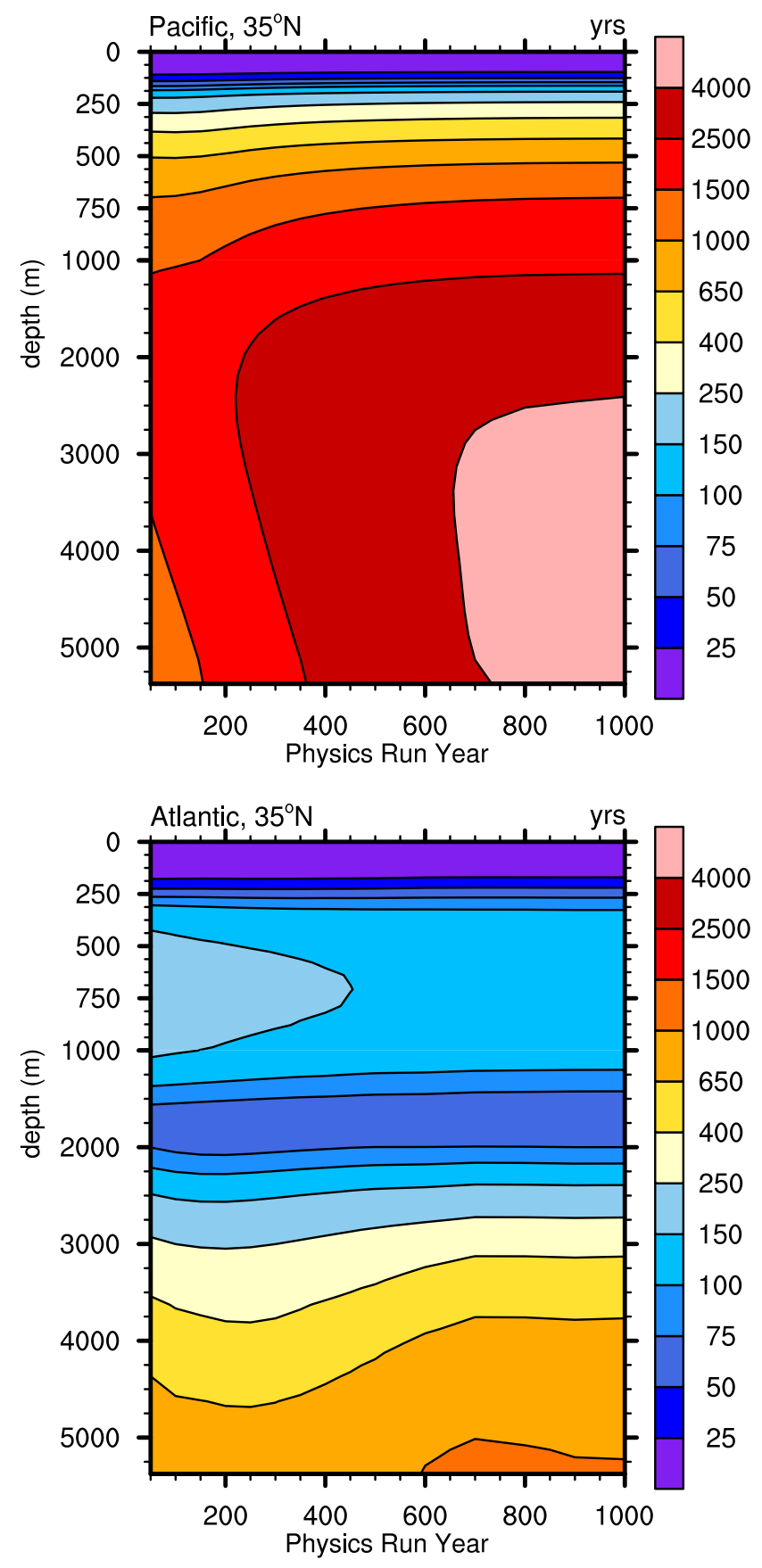

Figure 4: Zonal mean of spun-up ideal age at $35^{\circ} \mathrm{N}$ as function of physics run year used in the spin-up of ideal age: (a) Pacific basing (b) Atlantic basin. 

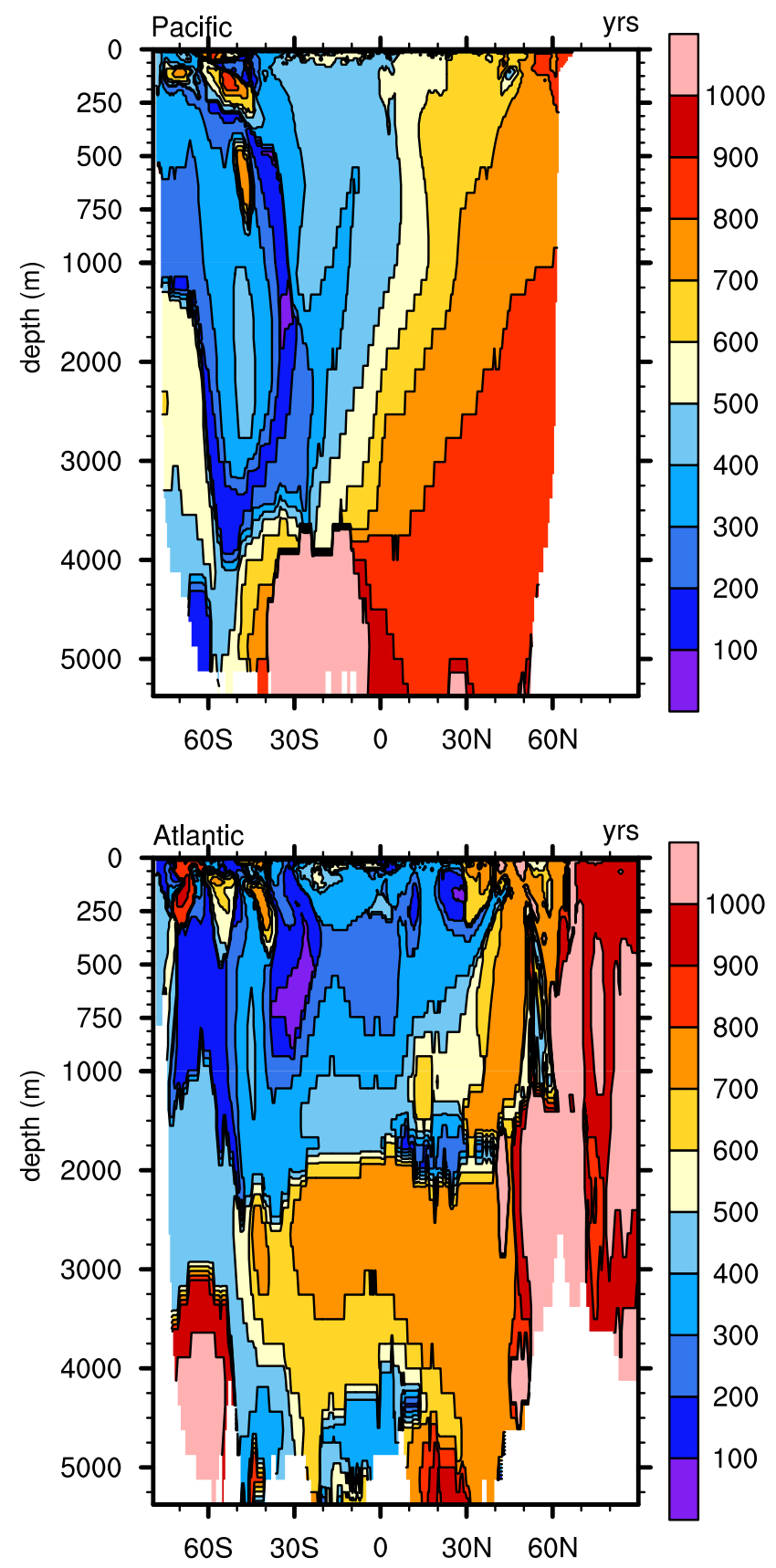

Figure 5: Duration of physics run needed to ensure that relative drift in the zonal mean of the spun-up ideal age is below the threshold of $2 \cdot 10^{-4} \mathrm{yrs} / \mathrm{yr}^{2}$ : (a) Pacific basin, (b) Atlantic basin. 


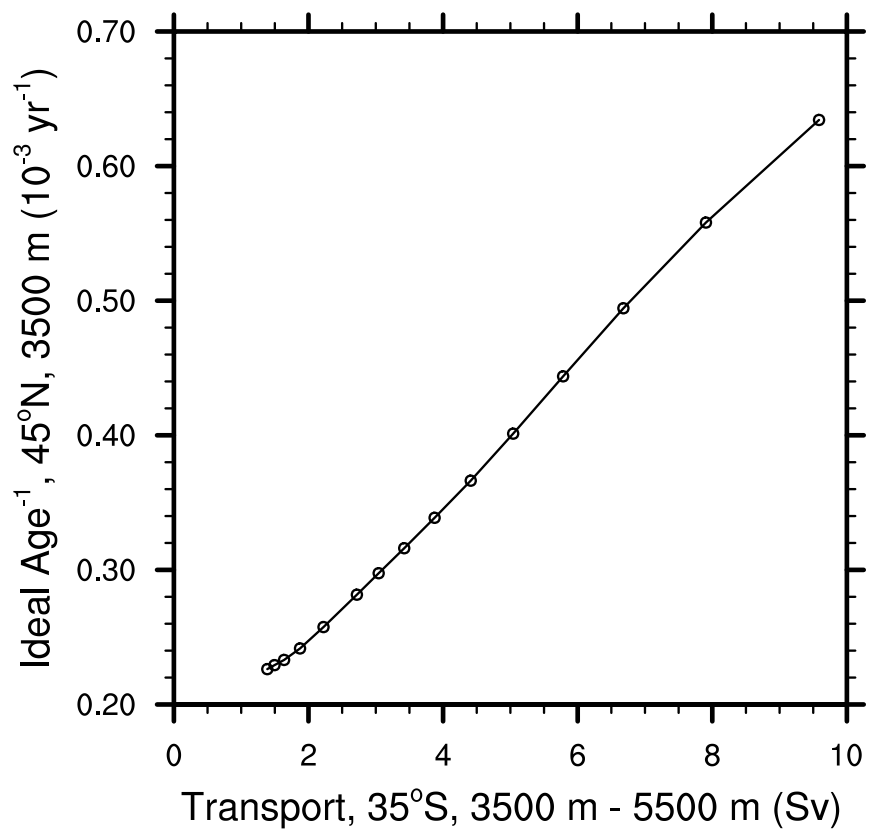

Figure 6: Inverse of zonal mean across the Pacific of spun-up ideal age at $45^{\circ} \mathrm{N}$ and depth of $3500 \mathrm{~m}$ vs. northward volumetric flux (Eulerian and bolus) integrated from $3500 \mathrm{~m}$ to $5500 \mathrm{~m}$ across the Pacific at $35^{\circ} \mathrm{S}$. 
tion over longer timescales. If the sampled circulation is not representative, and is anomalous in some way (e.g., deep water formation is uncharacteristically high), then this anomalous circulation is reflected in the spun-up tracers. We have found that if the sampled circulation is long enough, and hence representative, then the solution produced by the Newton-Krylov solver is useful in the sense that tracer drift in simulations initialized with the corresponding spun-up tracers is small. An alternative approach to handling non-periodic circulation is to modify Eq. (3) in a way that reflects the non-periodic nature of the circulation (e.g., to compute the end-state minus start-state tracer difference in water mass coordinates instead of Eulerian coordinates). We have not pursued such alternative approaches to extend the solver to non-periodic circulation.

\subsection{Stability of Simulations Initialized with Spun-up Tracers}

In Section 3.2, we showed that tracer drift is small in simulations initialized with tracers that were spun up using the Newton-Krylov solver. In simulations repeatedly using the same circulation that was used in the Newton-Krylov solver, tracer drift remained similar to the Newton-Krylov residuals. From this, and the convergence results in Section 3.1, we conclude that the Newton-Krylov solver is working properly. In simulations using a circulation that evolved away from the circulation used in the Newton-Krylov solver, tracer drift was larger and essentially independent of how converged the Newton-Krylov solution was. We conclude that the additional drift, above the Newton-Krylov residuals, is due to the changes in the freely evolving circulation. We glean two lessons from these results. The first is that when applying the Newton-Krylov solver to spin up tracers for initializing 
simulations, it is important to use a circulation field in the solver iterations that is representative of the circulation of the simulations being initialized. For example, the change in circulation over years 250 to 260 with NY forcing was sufficient to induce non-trivial change in ideal age. So if one's objective were to have minimal change in ideal age over that 10-year period, it would be better to use the Newton-Krylov solver with $\Delta t=10$ years. As alluded to in Section 4.1, this point is particularly relevant for circulation fields with interannual variability, that it is important to use a representative period of circulation in that situation. The second lesson is that if the circulation field used in the solver does differ from the circulation that is ideal for the application at hand, one need not choose a solver convergence criterion that is tighter than the anticipated drift from the mismatch in circulation.

\subsection{Computational Performance}

We documented, in Section 3.3, the substantial performance benefit that the Newton-Krylov offers compared to the brute force spin-up approach. However, it is fair to point out that if the ocean model's circulation field has interannual variability, then these speed-up factors decrease, because the cost of evaluating $\boldsymbol{G}$ increases. That said, the cost of solving the system of linear equations related to the preconditioner would not change. If evaluating $G$ involved running the ocean model 10 years, and one had a good initial Newton iterate, then we estimate that our Newton-Krylov solver would be faster than the brute force spin-up approach by a factor of over 100 . 


\subsection{Dependence of Spun-up Tracer Concentrations on Duration of Physics} Run

The results in Section 3.4 demonstrate that it may be necessary to run the physics run many centuries in order to ensure robust application of the Newton-Krylov solver for tracers. While these results are disappointing, it is worth noting that the physics run need not include the tracers to which the Newton-Krylov solver is applied. For tracer modules with many tracers, this yields substantial savings in the computational cost in the physics run. Moreover, we hypothesize that the weak ventilation bias in our ocean model's circulation may be increasing the required duration of the physics run. The results for ideal age in Section 3.4 and natural abiotic radiocarbon, not shown, indicate that the deep overturning circulation in the Pacific is sluggish early in the physics run. We hypothesize that this anemic ventilation causes the model predicted density structure to drift away from observations, which in turn further reduces the ventilation, and that this feedback contributes to the long duration that is required for the physics run in order to achieve robust spun-up tracers. A prediction that arises from this hypothesis is that if the model parameterizations were to achieve more realistic ventilation, then the physics run would not need to be so long in order to achieve robust spun-up tracer concentrations.

Another approach to reduce the computational time for the physics run is to extend the Newton-Krylov solver to the active tracers of the ocean model. While we have not done this, we speculate that the main challenge that would arise in directly applying the technique to the active tracers would be constructing a preconditioner that is both feasible to invert and adequately 
represents how the model's circulation, advection and mixing, depends upon the active tracers. We note that Bernsen et al. (2008) have applied NewtonKrylov solvers to a spin up a geostrophic ocean model, and Bernsen et al. (2010) have done so for a simplified configuration of version 4 of the Modular Ocean Model (MOM4).

\subsection{Implementing the Solver in Different Ocean Models}

It is of practical interest to apply the Newton-Krylov solver that we have developed to other ocean models (e.g., besides POP2). We first point out the advice of Kellev (2003), that it is preferable to use an existing Newton-Krylov implementation instead of implementing your own. That said, some batch computing environments, such as ours, make this impractical. The following is intended as a guide for modellers in this situation. We briefly describe, from a bottom-up perspective, the tools that are necessary to implement the solver. These tools are shell scripts in our implementation, but any implementation that achieves the required functionality would suffice. We initially describe the tools needed for a non-preconditioned Newton-Krylov solver, and then separately describe the tools for the Krylov preconditioner. At the lowest level, the Newton-Krylov solver requires a tool to compute $\boldsymbol{G}\left(\boldsymbol{c}\left(t_{0}\right)\right)$, Eq. (3)), for an arbitrary initial tracer state $\boldsymbol{c}\left(t_{0}\right)$. Computing $\boldsymbol{G}$ involves running the model forward in time for duration $\Delta t$, and computing the difference between the tracer states $\boldsymbol{c}\left(t_{0}+\Delta t\right)$ and $\boldsymbol{c}\left(t_{0}\right)$. The details of how to do this will vary for different ocean models and computing environments. The next requirement is a tool to apply Eq. (10) to compute the matrix-vector multiplications required by GMRES. With these two tools, one can follow Algorithm 9.4 of Saad (2003) to implement GMRES to solve for the increment 
in Newton's method, $\delta \boldsymbol{c}_{n}$. Then one applies Eqs. (7) and (A.1) to implement Newton's method to solve for the spun-up tracers.

Computing matrix-vector multiplications with the preconditioner matrix involves solving a system of linear equation with the matrix $\boldsymbol{Q}=\Delta t \partial \overline{\boldsymbol{f}} / \partial \boldsymbol{c}$. The description of the construction of the $\boldsymbol{Q}$ in Section 2.3 is in general applicable to other ocean models. The primary tool necessary for this construction is an implementation of the IR tracers. It is also necessary to be able to generate diagnostic output of tracer tendencies for the IR tracers. We expect that it is straightforward for a knowledgable modeller to implement such a tracer module in an ocean model that has flexible support for tracers. In order for the IR tracer approach to be practical, it is necessary to come up with a decomposition of the set of the model's grid points into subsets such that each IR tracer from a given subset can be superimposed without their tendencies overlapping. We have done this for POP's structured grid by superimposing IR tracers whose grid indices are offset from each other with a regularly spaced offset that is greater than POP's operator's stencil width. We expect that a more involved approach would be necessary for an ocean model on an unstructured grid. The IR tracer approach can be used to compute the terms of $\boldsymbol{Q}$ that are related to the ocean model's circulation, using the diagnostic IR tracer tendencies and Eq. (19). A technical aspect of this process is mapping the IR tracer tendencies to the correct entry in the data structure of the sparse matrix. In order to do this, we first construct mappings between the model's grid index space and the index space of the sparse matrix. The location of non-zero entries in $\boldsymbol{Q}$ are then given by the composition of these index mappings with the model's grid cell adjacency 
matrix. For structured grids, non-zero entries in this latter matrix correspond to offsets in the model's grid index space. For unstructured grids, we presume that this latter matrix would be provided along with the model grid. The terms of $\boldsymbol{Q}$ that arise from tracer source-sink terms are more a function of the tracer dynamics than the underlying ocean model. The approaches we described in Section 2.3 for these terms are applicable to general ocean models.

\subsection{Benefits of the Brute Force Spin-up Approach}

We note that there can be useful information in the transient tracer solution generated using the brute force spin-up approach. For example, for ideal age with steady circulation, the transient tracer solution can be used to derive the boundary propagator (Hall and Haine, 2002). This information is not generated when the Newton-Krylov solver is used to directly solve for the spun-up tracers. For applications where this information is of interest, the Newton-Krylov solver presented here would not be an appropriate tool.

\section{Conclusions}

We have presented a Newton-Krylov solver to spin up tracers in an ocean model. That is, it solves Eq. (3) using Eqs. (6) and (17). We have shown that the Newton-Krylov solver converges to a solution of Eq. (3), that free running simulations initialized with the resulting spun-up tracer have small drift, and that the solver produces these spun-up tracers with orders of magnitude less computational time than the brute force spin-up approach. This demonstrates that the solver can be an effective tool for fast spin-up of ocean tracers. 
We have also used the solver to spin up ideal age and natural abiotic radiocarbon to initialize coupled model simulations with CESM and to evaluate parameterizations being considered for version 2 of CESM. We are actively working on extending the solver to biogeochemical tracer modules, where non-linearities in tracer source-sink terms necessitate enhancements to the solver. Beyond biogeochemical tracers, we plan to extend the solver to the ocean model's active tracers, temperature and salinity.

\section{Appendix A. Description of the Newton-Krylov Solver Implemen- tation}

Quality implementations of Newton-Krylov solvers are available. For example, Kelley's nsoli.m (Kelley, 2003) is an implementation in MATLAB, and KINSOL (Collier et al., 2015) is a distributed memory parallel implementation applicable to larger problems. However, to the best of our knowledge, these implementations, and others that we are aware of, require that the solver remain in memory for the duration of the solve. This is problematic in our batch computing environment for the following reasons. Evaluating $\boldsymbol{G}$, the function whose root we are trying to find, for the model configuration described in Section 2.2 , takes $\approx 1 / 2$ an hour for the ideal age tracer and $\Delta t=1$ year. This evaluation time increases linearly with increasing $\Delta t$. It also increases with increasing tracer count, i.e., when there is more than one tracer being simultaneously spun up. In our batch computing environment, large-scale computing jobs have a wall-clock limit of 12 hours. This places a restrictive upper limit on how many times $\boldsymbol{G}$ can be evaluated in a single job submission. For example, if one were spinning up the ideal age tracer 
with respect to a circulation field that has interannual variability, and used $\Delta t=8$ years, then only $3 \boldsymbol{G}$ evaluations would be possible in a single batch submission. This makes it impractical to use one of these existing solvers out of the box.

We have instead implemented our own Newton-Krylov solver. In our implementation, we have split the solver into small callable pieces whose individual run times fit within the constraints of our batch computing environment. A light-weight driver, which can run outside of the wall-clock restrictions, manages the calling of the components of the solver. Quantities that would persist in memory in the out-of-the-box solvers, such as basis vectors of the Krylov solver, function evaluations, and intermediate coefficients from the solver, are stored in files.

Our implementation of Newton's method is fairly simplistic. We compute the Newton increment $\delta \boldsymbol{c}_{n}$, defined by Eq. ([6), using the Krylov solver. Then, following (Kelley, 2003, Section 1.6), we find the smallest integer $m \geq 0$ such that

$$
\left\|\boldsymbol{G}\left(\boldsymbol{c}_{n}+2^{-m} \delta \boldsymbol{c}_{n}\right)\right\|<\left(1-\alpha 2^{-m}\right)\left\|\boldsymbol{G}\left(\boldsymbol{c}_{n}\right)\right\|,
$$

where $\|\cdot\|$ is the volume weighted root-mean-square norm, and set $\boldsymbol{c}_{n+1}=$ $\boldsymbol{c}_{n}+2^{-m} \delta \boldsymbol{c}_{n}$. This condition, Eq. (A.1), referred to by Kelley (2003) as the Armijo rule, ensures that the solver is making a sufficient decrease in $\|\boldsymbol{G}\|$. Following Kelley (2003), we use $\alpha=10^{-4}$. We have used this simple line search method instead of one of the more sophisticated methods described in Kelley (2003) because of the relatively high computational cost of evaluating our $\boldsymbol{G}$.

Our implementation of preconditioned GMRES follows Algorithm 9.4 of 
Saad (2003). Dot products of vectors are used throughout GMRES (e.g., to compute projections and to normalize vectors). Consistent with $\|\cdot\|$ being the volume weighted root-mean-square norm, dot products of vectors of tracer concentrations are defined as volume weighted sums of tracer products. In our application of Eq. (10) to compute the matrix-vector multiplications required by GMRES, we normalize $\|\delta \boldsymbol{c}\|=1$ and set $\sigma=.5 \cdot 10^{-3}$. We have not investigated the sensitivity of the solver to the selection of $\sigma$. It is possible that tracers with more complicated dynamics than ideal age will require a more refined approach for the selection of $\sigma$, such as the method described in (Kelley, 2003, Section 3.2.1).

\section{Appendix B. Derivation of Krylov Preconditioner}

We present here the differential and algebraic manipulations that lead from Eq. (13) to Eq. (14). Differentiating both sides of Eq. (13) with respect to $\boldsymbol{c}\left(t_{0}\right)$ yields

$$
\frac{\partial \tilde{\boldsymbol{\Phi}}}{\partial \boldsymbol{c}\left(t_{0}\right)}-\boldsymbol{I}=\Delta t \frac{\partial \overline{\boldsymbol{f}}}{\partial \boldsymbol{c}} \frac{\partial \tilde{\boldsymbol{\Phi}}}{\partial \boldsymbol{c}\left(t_{0}\right)},
$$

${ }_{694}$ where $\boldsymbol{I}$ is the identity matrix. Rearranging this to solve for $\partial \tilde{\boldsymbol{\Phi}} / \partial \boldsymbol{c}\left(t_{0}\right)$ yields

$$
\frac{\partial \tilde{\boldsymbol{\Phi}}}{\partial \boldsymbol{c}\left(t_{0}\right)}=\left(\boldsymbol{I}-\Delta t \frac{\partial \overline{\boldsymbol{f}}}{\partial \boldsymbol{c}}\right)^{-1}
$$

This enables the following algebraic manipulations to derive Eq. (14):

$$
\begin{aligned}
\boldsymbol{P}_{n} & =\tilde{\boldsymbol{J}}^{-1}=\left(\frac{\partial \tilde{\boldsymbol{G}}}{\partial \boldsymbol{c}\left(t_{0}\right)}\right)^{-1}=\left(\frac{\partial \tilde{\boldsymbol{\Phi}}}{\partial \boldsymbol{c}\left(t_{0}\right)}-\boldsymbol{I}\right)^{-1} \\
& =\left(\left(\boldsymbol{I}-\Delta t \frac{\partial \overline{\boldsymbol{f}}}{\partial \boldsymbol{c}}\right)^{-1}-\boldsymbol{I}\right)^{-1}=\left(\Delta t \frac{\partial \overline{\boldsymbol{f}}}{\partial \boldsymbol{c}}\right)^{-1}-\boldsymbol{I}
\end{aligned}
$$


where we have made use of the following chain of matrix identities:

$$
\begin{aligned}
& \left((\boldsymbol{I}-\boldsymbol{M})^{-1}-\boldsymbol{I}\right)^{-1}=\left((\boldsymbol{I}-\boldsymbol{M})^{-1}-(\boldsymbol{I}-\boldsymbol{M})(\boldsymbol{I}-\boldsymbol{M})^{-1}\right)^{-1} \\
& \quad=\left(\boldsymbol{M}(\boldsymbol{I}-\boldsymbol{M})^{-1}\right)^{-1}=(\boldsymbol{I}-\boldsymbol{M}) \boldsymbol{M}^{-1}=\boldsymbol{M}^{-1}-\boldsymbol{I}
\end{aligned}
$$

\section{Appendix C. Utilizing SuperLU_DIST}

As stated in Section 2.3. once we construct the matrix $\boldsymbol{Q}=\Delta t \partial \overline{\boldsymbol{f}} / \partial \boldsymbol{c}$, we use SuperLU_DIST to solve the system of linear equations in Eq. (16). As its name suggests, SuperLU_DIST does this by first constructing an LU decomposition of the matrix $\boldsymbol{Q}$ and then using forward and backward substitution to directly solve the factored systems of linear equations. The DIST suffix denotes that we are using the distributed memory version of the library. We are using the parallel symbolic factorization functionality in SuperLU_DIST (Grigori et al., 2007). Compared to the serial version, the parallel version significantly reduces the time and memory usage of the symbolic factorization step of constructing the LU decomposition. A drawback of our batch computing environment is that the queue wall-clock limits preclude us from keeping the LU decomposition in memory, once it is constructed. Because SuperLU_DIST does not have the ability to store the LU decomposition to disk, we end up recreating it every iteration of the Krylov solver. Using a sparse matrix library that has the ability to store the factored matrix to disk would perhaps improve the overall computational efficiency of our NewtonKrylov solver. 


\section{Acknowledgments}

The National Center for Atmospheric Research is sponsored by the National Science Foundation. This work was supported in part by the Department of Energy, Office of Science, Biological and Environmental Research, under SciDAC award number DE-SC0006768. Computing resources were provided by the Climate Simulation Laboratory at NCAR's Computational and Information Systems Laboratory (CISL), sponsored by the National Science Foundation and other agencies. The CESM project is supported by the National Science Foundation and the Office of Science (BER) of the U.S. Department of Energy. We thank François Primeau, Matthew Long, Frank Bryan, and three anonymous reviewers for their constructive comments.

\section{References}

Bardin, A., Primeau, F., Lindsay, K., 2014. An offline implicit solver for simulating prebomb radiocarbon. Ocean Model. 73, 45-58.

Bardin, A., Primeau, F., Lindsay, K., Bradley, A., 2016. Evaluation of the accuracy of an offline seasonally-varying matrix transport model for simulating ideal age. Ocean Model. 105, 25-33.

Bernsen, E., Dijkstra, H. A., Thies, J., Wubs, F. W., 2010. The application of Jacobian-free Newton-Krylov methods to reduce the spin-up time of ocean general circulation models. J. Comput. Phys. 229 (21), 8167-8179.

Bernsen, E., Dijkstra, H. A., Wubs, F. W., 2008. A method to reduce the spin-up time of ocean models. Ocean Model. 20 (4), 380-392. 
Collier, A. M., Hindmarsh, A. C., Serban, R., Woodward, C. S., July 2015. User documentation for KINSOL v2.8.2. Tech. Rep. UCRL-SM-208116, Center for Applied Scientific Computing, Lawrence Livermore National Laboratory, http://computation.llnl.gov/casc/sundials/documentation/kin_guide.pdf.

Danabasoglu, G., Bates, S. C., Briegleb, B. P., Jayne, S. R., Jochum, M., Large, W. G., Peacock, S., Yeager, S. G., 2012. The CCSM4 ocean component. J. Climate 25 (5), 1361-1389.

Danabasoglu, G., Large, W. G., Briegleb, B. P., 2010. Climate impacts of parameterized Nordic Sea overflows. J. Geophys. Res. 115 (C11).

DeVries, T., Primeau, F., 2011. Dynamically and observationally constrained estimates of water-mass distributions and ages in the global ocean. J. Phys. Oceanogr. 41 (12), 2381-2401.

Gent, P. R., McWilliams, J. C., 1990. Isopycnal mixing in ocean circulation models. J. Phys. Oceanogr. 20 (1), 150-155.

Grigori, L., Demmel, J. W., Li, X. S., 2007. Parallel symbolic factorization for sparse LU with static pivoting. SIAM J. Sci. Comput. 29 (3), 1289-1314.

Hall, T. M., Haine, T. W. N., 2002. On ocean transport diagnostics: The idealized age tracer and the age spectrum. J. Phys. Oceanogr. 32 (6), $1987-$ 1991.

Holland, M. M., Bailey, D. A., Briegleb, B. P., Light, B., Hunke, E., 2012. Improved sea ice shortwave radiation physics in CCSM4: The impact of melt ponds and aerosols on Arctic sea ice. J. Climate 25 (5), 1413-1430. 
Holland, W. R., Chow, J. C., Bryan, F. O., 1998. Application of a third-order upwind scheme in the NCAR ocean model. J. Climate 11 (6), 1487-1493.

Holzer, M., Primeau, F. W., 2010. Improved constraints on transit time distributions from argon 39: A maximum entropy approach. J. Geophys. Res. 115 (C12).

Hunke, E. C., Lipscomb, W. H., 2008. CICE: The Los Alamos Sea Ice Model, documentation and software user's manual, version 4.0. Tech. Rep. LA-CC06-012, Los Alamos National Laboratory, Los Alamos, NM.

Hurrell, J. W., Holland, M. M., Gent, P. R., Ghan, S., Kay, J. E., Kushner, P. J., Lamarque, J.-F., Large, W. G., Lawrence, D., Lindsay, K., Lipscomb, W. H., Long, M. C., Mahowald, N., Marsh, D. R., Neale, R. B., Rasch, P., Vavrus, S., Vertenstein, M., Bader, D., Collins, W. D., Hack, J. J., Kiehl, J., , Marshall, S., 2013. The Community Earth System Model: A framework for collaborative research. Bull. Amer. Meteor. Soc. 94 (9), 1339-1360.

Kelley, C. T., 2003. Solving Nonlinear Equations with Newton's Method. Society for Industrial and Applied Mathematics, Philadelphia, PA.

Khatiwala, S., 2008. Fast spin up of ocean biogeochemical models using matrix-free Newton-Krylov. Ocean Model. 23 (3-4), 121-129.

Khatiwala, S., Primeau, F., Holzer, M., 2012. Ventilation of the deep ocean constrained with tracer observations and implications for radiocarbon estimates of ideal mean age. Earth Planet. Sci. Lett. 325-326, 116-215. 
Khatiwala, S., Visbeck, M., Cane, M. A., 2005. Accelerated simulation of passive tracers in ocean circulation models. Ocean Model. 9 (1), 51-69.

Large, W. G., McWilliams, J. C., Doney, S. C., 1994. Oceanic vertical mixing: A review and a model with a nonlocal boundary layer parameterization. Rev. Geophys. 32 (4), 363-403.

Large, W. G., Yeager, S. G., 2004. Diurnal to decadal global forcing for ocean and sea-ice models: The data sets and flux climatologies. Tech. Rep. TN460+STR, NCAR.

Levitus, S., Boyer, T., Concright, M., Johnson, D., O’Brien, T., Antonov, J., Stephens, C., Garfield, R., 1998. World Ocean Database 1998, Volume 1: Introduction. NOAA Atlas NESDIS 18, U.S. Government Printing Office, Washington, D.C., p. 346.

Li, X., Primeau, F. W., 2008. A fast Newton-Krylov solver for seasonally varying global ocean biogeochemistry models. Ocean Model. 23 (1-2), 1320.

Li, X. S., Demmel, J. W., June 2003. SuperLU_DIST: A scalable distributedmemory sparse direct solver for unsymmetric linear systems. ACM Trans. Math. Software 29 (2), 110-140.

Li, X. S., Demmel, J. W., Gilbert, J. R., Grigori, L., Shao, M., Yamazaki, I., September 1999. SuperLU Users' Guide. Tech. Rep. LBNL-44289, Lawrence Berkeley National Laboratory, http://crd.lbl.gov/ xiaoye/SuperLU/. 
Orr, J., Najjar, R., Sabine, C., Joos, F., 2000. Abiotic-HOWTO. Internal OCMIP report, LSCE/CEA Saclay, Gif-sur-Yvette, France, http://ocmip5.ipsl.jussieu.fr/OCMIP/phase2/simulations/Abiotic/HOWTO-Abiotic.htn

Saad, Y., 2003. Iterative Methods for Sparse Linear Systems, 2nd Edition. Society for Industrial and Applied Mathematics, Philadelphia, PA.

Saad, Y., Schultz, M. H., 1986. GMRES: A generalized minimal residual algorithm for solving nonsymmetric linear systems. SIAM J. Sci. and Stat. Comput. 7 (3), 856-869.

Smith, R., Jones, P., Briegleb, B., Bryan, F., Danabasoglu, G., Dennis, J., Dukowicz, J., Eden, C., Fox-Kemper, B., Gent, P., Hecht, M., Jayne, S., Jochum, M., Large, W., Lindsay, K., Maltrud, M., Norton, N., Peacock, S., Vertenstein, M., Yeager, S., 2010. The Parallel Ocean Program (POP) reference manual, ocean component of the Community Climate System Model (CCSM). Tech. Rep. LAUR-10-01853, Los Alamos National Laboratory, Los Alamos, NM, http://www. cesm.ucar.edu/models/cesm1.0/pop2/doc/sci/POPRefManual.pdf.

Steele, M., Morley, R., Ermold, W., 2001. PHC: A global ocean hydrography with a high-quality Arctic Ocean. J. Climate 14 (9), 2079-2087.

Thiele, G., Sarmiento, J. L., 1990. Tracer dating and ocean ventilation. J. Geophys. Res. 95 (C6), 9377-9391. 